\title{
NEW DATA ON THE SOLAR SUPERCORONA
}

\author{
v. V. VITKEVICH \\ Academy of Sciences, Moscow, U.S.S.R.
}

1. This paper reports on the results of the investigation of the outermost regions of the solar corona (which for brevity I shall call the supercorona) that have been obtained during recent years.

Seven years ago, in 1951, a new method of observation was published in Doklady of the Academy of Sciences of the U.S.S.R. [1, 2]. The idea of the method, which at the present time is well known, is that the radio emission of the Crab nebula is received at the time when the source is covered by the solar corona. This original eclipse takes place yearly in the middle of June.

By receiving the radio waves that pass through the solar corona regions, we can estimate the effects of attenuation, scattering and refraction and reach some conclusions about the structure of the supercorona.

2. The first observations that gave results were carried out in 1951 in the Crimea with the aid of the sea interferometer at a 4-meter wavelength. The intense radio-spot that appeared on the sun seemed to exclude the possibility of obtaining results.

Beginning on June 18, however, when the rising of the radio source was observed considerably earlier than that of the sun, the intensity of the Crab nebula could be measured. On June 18 and 19 the amplitude of the interference lobes appeared to be less than on other days. At that time it was difficult to be sure that the phenomenon was due to the influence of the supercorona. In the light of further results, however, it became clear that the scattering effect of radio waves on electron inhomogeneities was observed at that time (Fig. 1).

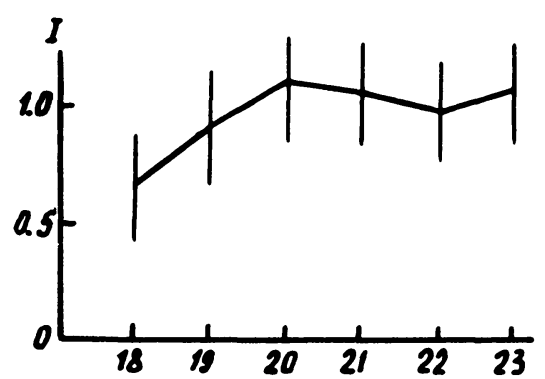

FIG. 1. First observations during occultation of Crab nebula by the solar corona (1951 June). $I=$ amplitude of lobes (sea interferometer).
The investigations of the Crimean Scientific Station (and of the Cavendish La. boratory independently) that were carried out during the following years gave these results: first, that the supercorona, in the range 4.5 to $20 R_{\odot}$, has an inhomogeneous structure $[3,4,5,6]$; second, that these inhomogeneities are stationary in the sense that scattering effects are observed yearly; third, that some asymmetry of inhomogeneities is established. The scattering effect during the second phase of the eclipse (source remote) is stronger 275 
than during the first phase (source near) in observations with the interference base in the east-west direction.

From these observations the characteristics of the scattering medium can be found by solving the corresponding integral equation. If we adopt some size of inhomogeneities $l_{c}=10^{4} \mathrm{~km}$, their electron concentrations have the values in equatorial and polar regions of the supercorona shown in Table I.

TABLE I

ELECTRON Densities OF 104-km INHOMOgeneities IN THE SUPERCoRONA

$\begin{array}{rrrrrrrr}r / R_{\odot} & \text { equatorial } & \text { polar } & \text { mean } & r / R_{\odot} & \text { equatorial } & \text { polar } & \text { mean } \\ 4 & 17700 & 14500 & 16500 & 11 & 7000 & 2400 & 4900 \\ 5 & 16200 & 12300 & 14500 & 12 & 6000 & 1800 & 4000 \\ 6 & 14600 & 12000 & 12600 & 13 & 5000 & 1400 & 3100 \\ 7 & 12700 & 7900 & 10700 & 14 & 4200 & 1100 & 2500 \\ 8 & 11100 & 6000 & 9000 & 15 & 3500 & 1000 & 1800 \\ 9 & 9700 & 4600 & 7500 & 16 & 2800 & 900 & 1400 \\ 10 & 8300 & 3400 & 6000 & 18 & 1700 & 800 & 1100\end{array}$

These data were obtained on the basis of observations made in 1954-55, and of supposing the inhomogeneities to be isotropic. But we shall show that they are not, and in the future some correction must be made.

3. Let us analyze one result of the observations, namely, the dependence of the angle of scattering on the wavelength. Based on the geometric optics approximation, the angle of dispersion must have a quadratic dependence on wavelength. Fig. 2 gives the results of observations and calculations made

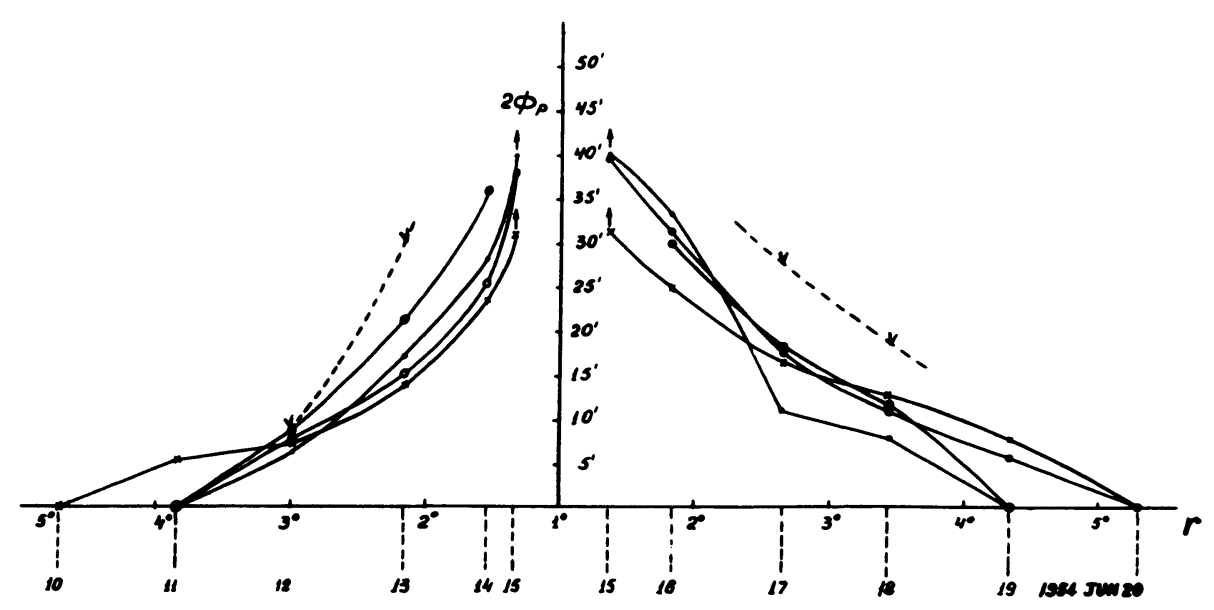

FIG. 2. $2 \Phi_{p}=$ the width of angular spectrum, $r=$ angular distance. The data of $1954: X X \lambda$ $=7.6 \mathrm{~m}, \Delta \phi=36^{\prime} ; \infty \lambda=5.8 \mathrm{~m}, \Delta \phi=27^{\prime} ; \bullet \bullet \lambda=5.8 \mathrm{~m}, \Delta \phi=37^{\prime} ; \odot \odot \lambda=3.5 \mathrm{~m}$, $\Delta \phi=23^{\prime} ; V V \lambda=2.74 \mathrm{~m}, \Delta \phi=23^{\prime}$. 
in 1954. The angle of dispersion $\emptyset_{p}$ stands for various wavelengths and various angular widths $\Delta \varphi$ of lobes. The figure shows the data on the angle of dispersion reduced to a $5.8 \mathrm{~m}$ wavelength (on the basis of the supposed quadratic dependence). Clearly, all the curves agree comparatively well, the scatter in almost all cases being attributable to inaccurate measurement. Fig. 3 shows the values reduced to a wavelength of $5.8 \mathrm{~m}$ for various wavelengths. Here the dependence upon wavelength is not observed. The greater values of $\Phi_{p}$ for $2.74 \mathrm{~m}$ are explained by the inaccuracy of observations, for the effect

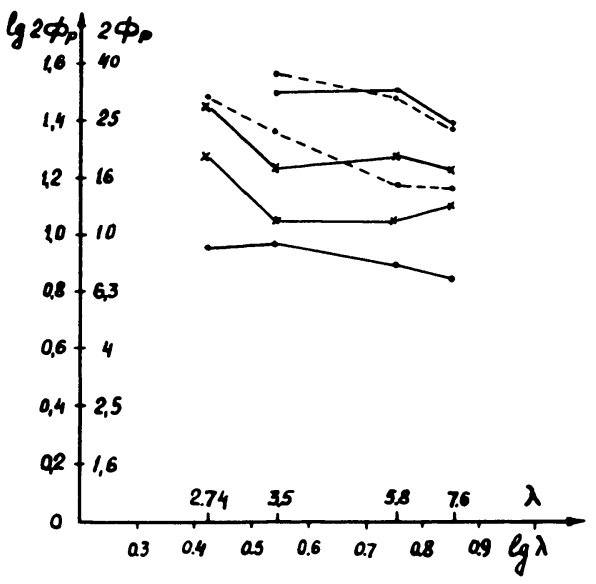

FIG. 3. Dependence of the angle of scattering $2 \Phi_{p}$ reduced to wavelength $5.8 \mathrm{~m}$ from wavelength of measuring, 1954. of scattering at this wavelength is comparatively small. Thus it may be considered that the dependence of $\boldsymbol{\Phi}_{p}$ upon wavelength is quadratic.

We can conclude that the optical depth $\tau$ for the longest wavelength is very small; in our case we get an optical depth $\tau \leq 0.1$ for $7.6 \mathrm{~m}$ wavelength. From the last value we can estimate the upper value of the temperature of the supercorona regions for $r \geq 1^{\circ} 40^{\prime}$.

If $\xi$ is the inhomogeneity coefficient defined by

$$
\xi=\frac{\overline{N_{e}^{2}}}{\left(\bar{N}_{e}\right)^{2}}
$$

then

$$
T_{e}^{3 / 2}>3.7 \times 10^{3} \xi ;
$$

Assuming $r \sim 7 R_{\odot}$ and $N_{e}=5 \times 10^{4}$, we find for $\xi=3, T_{e \text { min }}=5 \times 10^{4}$; if we accept $\xi=9$, then $T_{e m i n}=1.5 \times 10^{5}$. For $r=10 R_{\odot}, N_{e}=10^{4}$ and the values $T_{e \min }$ are correspondingly equal to $10^{4}$ and $3 \times 10^{4}$.

It should be noted that from the observations of 1954,1955 , and 1956 the values $\emptyset_{p}$ for the various sizes of interference bases for one and the same wavelength are the same. This result confirms once more the fact that the attenuation is very small. Since, in calculating the modulation depth, the law of radio-brightness distribution $\exp \left(-r^{2} / \Phi_{p}^{2}\right)$ is accepted, we come to the conclusion that this law does not contradict the observations.

4. From the observations made from 1951 to 1956 , and to 1958 we can establish the dependence of the size of the supercorona on the solar epoch. Such dependence is observed rather distinctly.

In Fig. 4 the sizes of the supercorona are given; the size $r_{1}$ is determined according to the first phase of eclipse, $r_{2}$ according to the second phase. The size $r_{1}+r_{2}$ is determined as the distance from the sun's center to the region 


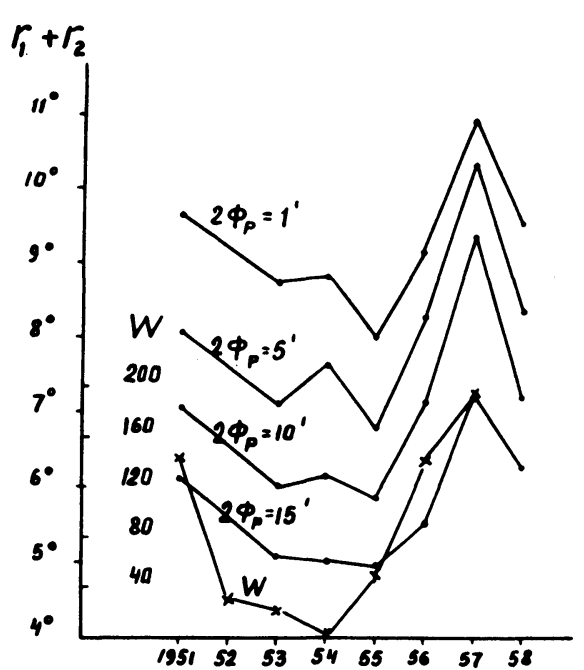

FIG. 4. The distances from the sun when the angle of scattering is equal to $2 \Phi^{\prime}$ (1951 to 1958). $W=$ Wolf's numbers. where the angle of dispersion has the value $2 \emptyset_{p}$. The values for the angle $2 \Phi_{p}$ are taken for $5.8 \mathrm{~m}$ wavelength according to the observations at that wavelength. It is clear that the size of the supercorona is associated with the phase of the 11-year period of solar activity; its size in 1957-58 is approximately 20 per cent greater than it was in 1953-55. The source of the origin of inhomogeneities, therefore, has to be found in solar activity. In the maximum year the matter is ejected from the sun more actively, which is perhaps the cause of the supercoronal inhomogeneities.

5. We come now to the subject of large inhomogeneities in the supercorona. The observations made in 1956 showed in three cases (on June 12,

13 , and 17) a remarkable refraction of radio waves, which shifted the whole interference pattern one to two interference lobes in comparison with the days out of the eclipse [7]. The phenomenon is explained by the presence of refraction of the order 0.5 to 1 degree in the solar corona.

The direction of the refraction is such that we can observe the apparent approach of the source to the sun, and estimate the density of electron inhomogeneities that are responsible for such refraction. If we suppose these inhomogeneities to be large-scale, about 2 to $4 R_{\odot}$ in diameter, and to extend as coronal rays, then it is necessary to have the electronic concentration $N_{e r}$ about 5 to $10 \times 10^{5}$.

Next we refer to the results of optical observations. It is known that coronal rays are approximately radial formations extending to many solar radii, and that their electron concentration, according to van de Hulst's data, can exceed the mean electron concentration of the corona 5 to 6 times. More exact calculations, however, made by G. M. Nikolskii [8] showed that the value could be increased to 10 .

Nikolskii's data on the 1954 June 30 eclipse show that the reduction of the electron concentration within the distance 4.5 to $7 R_{\odot}$ for the eastern ray is very inconsiderable, that the degree index is about 0.5 . The electron concentration of the ray at these distances is determined by the value $3.4 \times 10^{5}$. Iu. A. Nabudovich [9] concludes that the coronal rays within the range $r \geq 4 R_{\odot}$ can have a concentration 10 times larger than the spherically symmetric corona.

If we use the mean values of the electron concentration of the corona for distances 10 to $15 R_{\odot}$, then we get $N_{e}$ of the order of 7 to $2 \times 10^{4}$ [10]. Thus 
the ratio $N_{e r} / N_{e}=5$ to 15 is sensible. It corresponds closely to the data we got by an optical method.

Consequently, the results obtained do not contradict the existing theory on the nature of possible coronal inhomogeneities (in the form of coronal rays) at such remote distances, but they speak in favor of the possibility of their existence at the distance of the order 10 to $15 R_{\odot}$.

It should be noticed that in estimating the value $N_{e r}$ obtained by the radio method, we were considering the ideal case in which the ray was in the pattern plane and perpendicular to the direction of refraction. It is more probable that the ray has a radial direction to the sun; thus, it is necessary to take into account the corresponding factors.

6. At present there are definite indications that in explaining some of the experimental results additional considerations should be used. Some examples to this point:

(a) According to the author's previous observations [5], on 1953 June 20 at $3.5 \mathrm{~m}$ wavelength the intensity of the source under investigation increased by 80 per cent. Probably this increase was caused by the influence of the supercorona.

(b) According to Hewish [6], on 1953 June 10 at $3.7 \mathrm{~m}$ wavelength, as his diagrams show, the radiation intensity of the source decreased by 12.3 per cent, and on June 11 the intensity returned. On June 10 at $7.9 \mathrm{~m}$ wavelength the intensity of the source also decreased by 15.6 per cent (to our regret there are no observations on June 11).

(c) According to Slee's [11] observations of the source in 1956, with a onelobe antenna at a frequency of $85 \mathrm{Mc} / \mathrm{s}$, it was found that the intensity of the Crab nebula decreased by 30 per cent on the approach of the source to the sun at 7.5 and 11.2 solar radii. But there was detected no "expansion" of the source out of the range of the diagram ( \pm 1 degree).

(d) According to the French authors Blum and Boischot [12], at $1.77 \mathrm{~m}$ wavelength, when the width of the interference pattern lobe was 3.8 , on June 13 there was detected not a decrease but an increase of the whole intensity of the source by 59 per cent. Simultaneously widening of its size was observed. The refraction at that time did not exceed 30 seconds of arc.

(e) According to our data, there are cases when observations from two different bases or at two different wavelengths are incompatible.

As we see, there are cases in which the results of observations do not abide by the simple theory already given. In case $(a)$ the intensity increase is incomprehensible. In case $(b)$ it is not quite clear why on June 11 the intensity recovered; or more exactly, it is not clear why on June 10 the intensity decreased.

In case $(c)$ the intensity decrease and in case $(d)$ its increase are not clear. It seems possible to explain all the given examples if we admit (as we did in the previous paragraph) the existence in the supercorona of large electron inhomogeneities with sizes of the order of one or more solar radii.

By refracting radio waves these coronal rays can create noticeable changes 
(both increases and decreases) in the intensities of radio waves. Coronal rays are lenses for radio waves, focusing and defocusing them, with the result that the received energy changes essentially. Table II gives the results of some calculations.

As we can see, at rather considerable concentrations of the order of $10^{8}$ the refracting effect can be rather strong. The focal distance is less than 0.5 astronomical units, leading not to an increase but to a decrease of intensities. When $F$ is about one astronomical unit the intensity increase can be the biggest; as $F$ increases, the value of intensity gradually approaches 1. At electron concentrations in the "lens" less than $10^{4}$, in the range of metric waves it is impossible in reality to notice the change of intensity.

We examined an ideal case in which the incident wave is plane and the angular size of the source is very small. Considering the finite angular sizes

\section{TABLE II}

$\begin{array}{lllllll}N_{e r} & 10^{6} & 3 \times 10^{5} & 10^{5} & 3 \times 10^{4} & 10^{4} & 3 \times 10^{3} \\ 1-n & 1.5 \times 10^{-2} & 5 \times 10^{-3} & 1.5 \times 10^{-3} & 5 \times 10^{-4} & 1.5 \times 10^{-4} & 5 \times 10^{-5} \\ R & 200^{\prime} & 70^{\prime} & 20^{\prime} & 7^{\prime} & 2^{\prime} & 0.7 \\ F & 0.33 & 1.1 & 3.3 & 11 & 33 & 110 \\ I / I_{0} & 0.5 & 11 & 1.43 & 1.1 & 1.03 & 1.01\end{array}$

$N_{e r}=$ electron concentration of the ray which is supposed to be cylindrical, $2^{\circ}$ in diameter; $n=$ refractive index $(\lambda=5.8 \mathrm{~m}) ; R=$ radio refraction in minutes of arc; $\boldsymbol{F}=$ focal distance (in astronomical units); $\boldsymbol{I} / \boldsymbol{I}_{0}=$ relative values of intensity.

of the source, the change of the intensity of the flux will be less than the calculated one, but it will be large enough to explain the abnormalities mentioned above.

7. The irregularities can exist for a long time only in the presence of the magnetic field. The form of the inhomogeneities and the structure of the magnetic field may be isotropic or stretched. The result is that the effect of dispersion may be isotropic or anisotropic. Measurements with interferometers inclined at different angles to the axis of the sun were calculated $(1954,1957,1958)$. They showed that in different directions the value of scattering was not the same. (This is seen more clearly from the data of 1958.)

Two interferometers were used: the one with an east-west axis direction; the other with an approximate $30^{\circ}$ north-south axis. The results are presented in Fig. 5 (wavelength $5.8 \mathrm{~m}$ ), which shows that when the Crab nebula approaches the sun, the angles of scattering for two interferometers are essentially different; that the magnetic field, which determines the structure of inhomogeneities, is of a regular form; and that at the distances 28 to $15 R_{\odot}$ the magnetic field is nearly radial to the sun.

Near the sun the scattering is isotropic, from 5 to $10 R_{\odot}$. When the distance between the source and the sun increases, the value of scattering in both directions is the same. When the distance from the sun to the Crab 


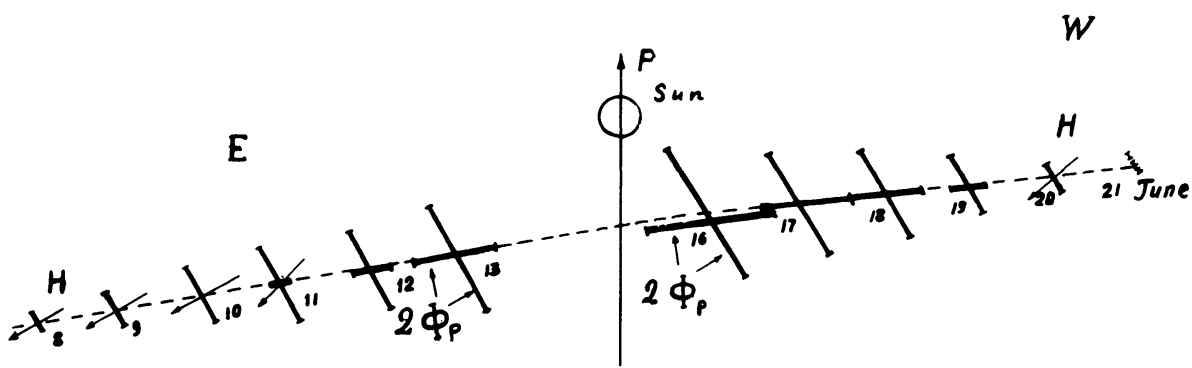

FIG. 5. The lengths of thick lines correspond to dimensions of angles of scattering; $H=$ the directions of magnetic field (data of 1958).

nebula decreases we observe the same effects. The direction of the magnetic field does not seem to change; it is the same as on the other side of the sun. If that is correct, the magnetic field is external to the sun.

It is clear from the observations that the inhomogeneities of the outer corona have a stretched structure. The magnetic field that determines their structure may have three components: the radial component of the magnetic field of the sun, a dipole component of the magnetic field of the sun, and the component that is external to the sun.

At the present time it is not possible to determine the values of these components, but it is necessary to continue the observations and to reach some conclusions. There is, however, no doubt that it is possible by using this method to get new results about the structure of the magnetic field at great distances from the sun. This is a very interesting and important problem.

\section{REFERENCES}

[1] Vitkevich, V. V. Dokl. Akad. Nauk S.S.S.R. 77, 585, 1951.

[2] Vitkevich, V. V. A. Zh. 29, 450, 1952.

[3] Vitkevich, V. V. Dokl. Akad. Nauk S.S.S.R. 101, 429, 1955.

[4] Vitkevich, V. V. A. Zh. 32, 150, 1955.

[5] Vitkevich, V. V. Transactions of the Fifth Conference on Cosmogonical Questions, Moscow (Acad. Sci. U.S.S.R. Press), 1956, p. 203.

[6] Hewish, A. Proc. Roy. Soc. A 228, 239, 1955.

[7] Vitkevich, V. V. A. Zh. 35, 52, 1958.

[8] Nikolskii, G. M. Dissertation, University of Kiev, 1955.

[9] Nadubovich, U. A. A. Zh. 33, 893, 1956.

[10] Blackwell, D. E. M.N.R.A.S. 116, 56, 1956.

[11] Slee, O. B. The Observatory, 76, 228, 1956.

[12] Blum, E. J., and Boischot, A. The Observatory, 77, 205, 1957.

[13] Vitkevich, V. V., and Panovkin, B. N. A. Z $h$. (In press.)

[14] Vitkevich, V. V. Proceedings of the Second Radioastronomical Conference in the U.S.S.R. (In press.) 